\title{
Kinematics of SNRs CTB 109 and $\mathrm{G} 206.9+2.3$
}

\author{
Margarita Rosado ${ }^{1}$, Mónica Sánchez-Cruces ${ }^{2}$ \\ and Patricia Ambrocio-Cruz ${ }^{3}$ \\ ${ }^{1}$ Instituto de Astronomía, UNAM, \\ Apartado Postal 70-264, Ciudad Universitaria 04510, \\ Ciudad de México, México \\ email: margarit@astro.unam.mx \\ ${ }^{2}$ ESFM-IPN, México, \\ email: \\ ${ }^{3}$ ITSOEH, México, \\ email: silviap@uaeh.edu.mx
}

\begin{abstract}
We present results of optical observations in the lines of $\mathrm{H} \alpha$ and [SII] ( $\lambda 6717$ and $6731 \AA$ A) obtained with the UNAM Scanning Fabry-Perot Interferometer PUMA (Rosado et al. 1995,RMxAASC, 3, 263 ) aimed at obtaining the kinematical distance, shock velocity and other important parameters of two supernova remnants (SNRs) with optical counterparts. We discuss on how kinematical distances thus obtained fit with other distance determinations. The studied SNRs are CTB 109 (SNR G109.1 - 1.0) hosting a magnetar (Sánchez-Cruces et al. 2017, in preparation) and the SNR G206.9+2.3 (Ambrocio-Cruz et al. 2014,RMxAA, 50, 323), a typical supernova remnant, to have a comparison. In Fig. 1 is depicted the [SII] line emission of two filaments of the optical counterpart of SNR CTB 109. We find complex radial velocity profiles obtained with the Fabry-Perot interferometer, revealing the presence of different velocity components. From these velocity profiles we obtain the kinematical distance, an expansion velocity of $188 \mathrm{~km} / \mathrm{s}$ and an initial energy of $8.1 \times 10^{50} \mathrm{ergs}$. These values are rather typical of other SNRs regardless that SNR CTB 109 hosts a magnetar. Thus, the mechanical energy delivered in the supernova explosion forming the magnetar does not seem to impact more than other $\mathrm{SNe}$ explosions the interstellar medium. This work has been funded by grants IN103116 and 253085 from DGAPA-UNAM and CONACYT, respectively.
\end{abstract}

Keywords. ISM: supernova remnants, ISM: kinematics and dynamics, shock waves.

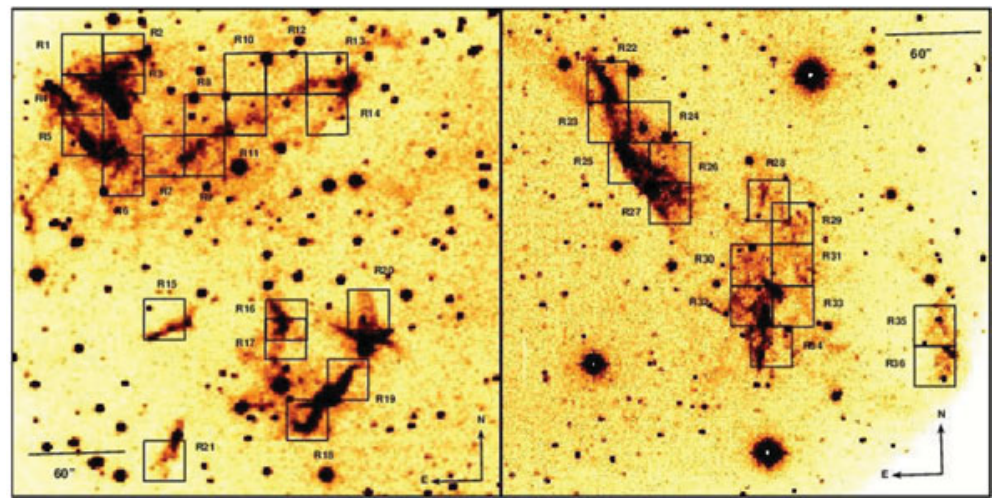

Figure 1. [SII] direct images showing the regions in which we divided the filaments in order to carry out our analysis (R1 to R36). Left and right panels correspond to the Northeastern and Southeastern filaments, respectively. 